\section{Altruism, Spite, and Greenbeards}

\section{Stuart A. West* and Andy Gardner*}

Hamilton's theory of inclusive fitness showed how natural selection could lead to behaviors that decrease the relative fitness of the actor and also either benefit (altruism) or harm (spite) other individuals. However, several fundamental issues in the evolution of altruism and spite have remained contentious. Here, we show how recent work has resolved three key debates, helping clarify how Hamilton's theoretical overview links to real-world examples, in organisms ranging from bacteria to humans: Is the evolution of extreme altruism, represented by the sterile workers of social insects, driven by genetics or ecology? Does spite really exist in nature? And, can altruism be favored between individuals who are not close kin but share a "greenbeard" gene for altruism?

$\mathrm{D}$ arwin's (1) theory of natural selection explains both the process and the purpose of adaptation (Fig. 1). The process is that heritable characters associated with greater reproductive success will be selected for and accumulate in natural populations. This leads to the apparent purpose of adaptation: characters appearing as if designed to maximize the individual's reproductive success (fitness).

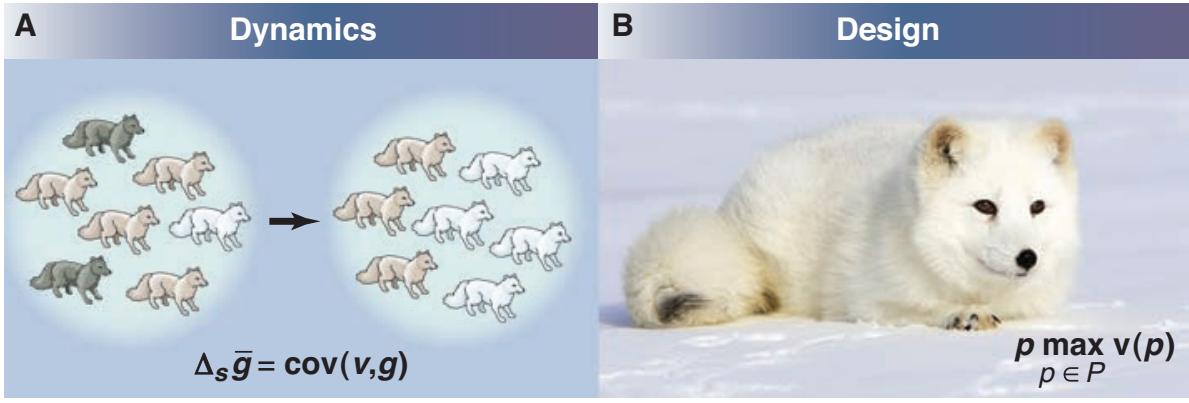

Fig. 1. Darwinian adaptation-dynamics and design. (A) Natural selection is the genetic change in the population owing to differential reproductive success of individual organisms. For example, among ancestral Arctic foxes, individuals with pale fur left more descendants than individuals with dark fur so that genes for pale fur accumulated in the population. Price's (35) equation formalizes this, stating that the change in the heritable portion of any character ascribable to natural selection $\left(\Delta_{s} \bar{g}\right)$ is equal to the covariance between an individual's genetic ("breeding") value for the trait $(g)$ and its relative fitness (v). (B) As a consequence of past natural selection, today's Arctic foxes look "designed" to fit their environment. This is captured by an optimization program, which states that the phenotype $(p)$ of the organism is functioning as if to maximize the individual's relative fitness ( $v$, more generally, its inclusive fitness) $(6,7)$. [Credit: Biosphoto/Cordier Sylvain/Peter Arnold Inc.]

Fisher (2) united Darwinism with Mendelian genetics by describing natural selection in terms of changes in gene frequencies. Genes associated with greater individual fitness are predicted to increase in frequency, leading to an increase in mean fitness. This "fundamental theorem of natural selection" was intended to capture the process (natural selection) and the purpose (maximization of individual fitness) of adaptation.

\section{Altruism, Spite, and Inclusive Fitness}

In the 1960s, Hamilton $(3,4)$ realized that maximization of individual fitness could not explain

Department of Zoology, Oxford University, South Parks Road, Oxford OX1 3PS, UK.

*To whom correspondence should be addressed. E-mail: stuart.west@zoo.ox.ac.uk (S.A.W); andy.gardner@zoo.ox. ac.uk (A.G.) reduces the reproductive success of the altruist — so why is it not weeded out by natural selection?

Hamilton (3) showed that genes can spread not only through their direct impact on their own transmission but also through their indirect impact on the transmission of copies present in other individuals. Consequently, altruistic behaviors can be favored if the benefits are directed toward other individuals who share genes for altruism (Fig. 2). This is encapsulated by Hamilton's rule (3), which states that a trait will be favored by selection when $r b-c>0$, where $c$ is the fitness cost to the actor, $b$ is the fitness benefit to the recipient, and $r$ is their genetic relatedness. This form of Hamilton's rule emphasizes interactions between two individuals, but it can be extended to allow for interactions with multiple individuals [supporting online material (SOM) text and table S1].
Hamilton's general point was that natural selection leads organisms to appear designed as if to maximize their inclusive fitness, which is the sum of fitness gained through producing offspring (direct fitness) and through affecting the fitness of related individuals (indirect fitness). The easiest way in which indirect fitness can be obtained is through interactions with close kin, in which case genes are identical by descent (from a common ancestor), and so this is usually referred to as "kin selection." However, inclusive fitness is not simply a concept that relates to interactions between relatives; it is our modern interpretation of Darwinian fitness, providing a general theory of adaptation $(6,7)$.

\section{Extreme Altruism and the Haplodiploidy Hypothesis}

The sterile workers of the social insects are extreme altruists, who give up any chance of independent reproduction in order to help others. In the Hymenoptera (ants, bees, and wasps) and termites, this extreme altruism has led to a division of labor between individuals and the evolution of the highest level of social organization, termed eusociality. Depending upon how eusociality is defined, it has evolved 3 to 11 times in the Hymenoptera, as well as in termites, thrips, aphids, spiders, beetles, shrimps, and mole rats $(8,9)$.

A major topic of debate has been whether eusociality has evolved multiple times in hymenopterans because of their haplodiploid genetics or their ecology. Under haplodiploidy, fertilized eggs develop into (diploid) females, and unfertilized eggs develop into (haploid) males. Hamilton (3) suggested that because haplodiploidy leads to a female being more related to her sisters $(r=0.75$, assuming an outbred population in which females mate once) than her own offspring $(r=0.5)$, this makes it easier for Hamilton's rule to be satisfied (Fig. 3A).

However, haplodiploidy also leads to a female being less related to a brother $(r=0.25)$, exactly canceling the benefit of increased relatedness to sisters (10). The haplodiploidy hypothesis is not rescued if the population sex ratio is biased toward females because this bias also reduces the relative reproductive value of females, exactly canceling any relatedness benefits (11). Numerous studies have examined whether certain life histories could rescue Hamilton's haplodiploidy hypothesis by making the average relatedness to siblings $r>0.5$, but none appears generally applicable (SOM text). In contrast, others have argued that what is special about the hymenopterans is that their natural history leads to a relatively high benefit of helping (high $b / c$ ratio), such as advanced parental care and a powerful sting that facilitates group defense (12).

\section{The Monogamy Hypothesis}

Recent work $(8,13)$ has suggested a possible resolution to this debate, arguing that strict lifetime monogamy, in which females only mate with 

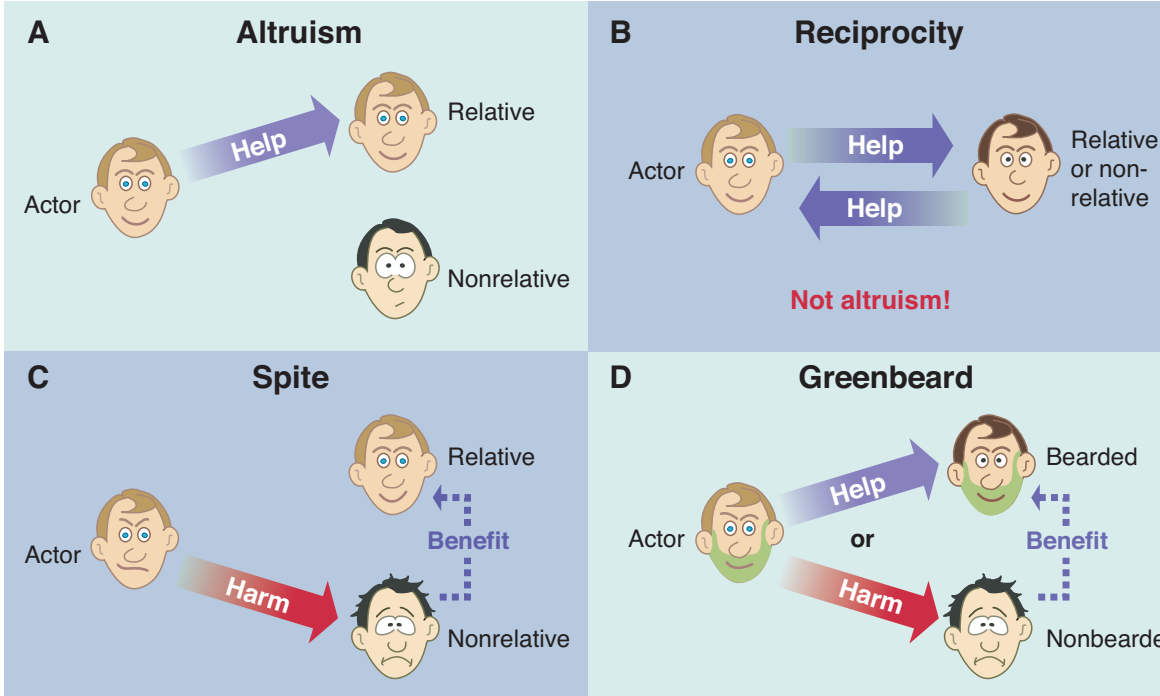

Not altruism!

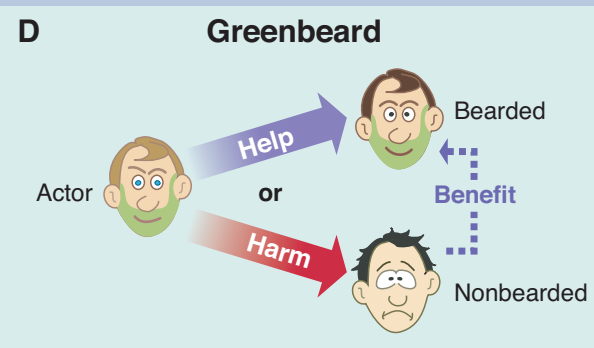

Fig. 2. Mechanisms for altruism, spite, and reciprocity. (A) Altruism can be favored if it is preferentially directed toward relatives. (B) Reciprocity is favored when it makes the actor more likely to receive help in the future. Reciprocity relies upon a future direct fitness benefit for cooperation and so is not altruistic. (C) Spite can be favored if it is preferentially directed toward relatively unrelated individuals, whose loss improves the fitness of relatives. (D) Greenbeards are favored by directing altruism toward fellow greenbeard individuals or spite toward nonbearded individuals.

one male in their entire life, is crucial for the evolution of eusociality. Monogamy leads to a potential worker being equally related $(r=0.5)$ to her own offspring and to the offspring of her mother (siblings). In this case, any small efficiency benefit for rearing siblings over their own offspring $(b / c>1)$ will favor eusociality (Fig. 3B).

In contrast, even a low probability of multiple mating means that potential workers would be more related to their own offspring. In this case, costly helping would require a significant efficiency advantage to rearing siblings over own offspring (Fig. 3C). Until group living is established, allowing the evolution of specialized cooperative behavior and division of labor, the ratio $b / c$ cannot be expected to greatly exceed 1 . For example, feeding a sibling is unlikely to be hugely more beneficial than feeding an offspring by the same amount. Consequently, in the absence of strict monogamy the population cannot even get started on the road to eusociality.

In support of this hypothesis, comparative studies have found monogamy to be the ancestral state in all the independent origins of eusociality studied $(9,14)$. Monogamy originated first, giving a high relatedness, and then when ecological conditions led to a high enough $b / c$, eusociality evolved (SOM text). Important ecological conditions include "life insurance" benefits of allowing helpers to complete parental care after the death of the mother (for example, ants, bees, and wasps), and "fortress defense" benefits of remaining to help use or defend a food source, when opportunities for successful migration are low (for example, aphids, beetles, termites, thrips, and shrimps) $(12,14)$.

There are some eusocial species in which queens mate multiply. However, this is a derived condition that has evolved after workers have already lost the ability to mate and realize full reproductive potential (9). Furthermore, these species have had the time to evolve division of labor and specialized helping behaviors, giving a substantial $b / c$.

The beauty of the monogamy hypothesis is that it simplifies our understanding of how eusociality evolved, emphasizing that the interaction between kinship and ecology is fundamental and that they are not competing explanations $(8,13)$. For example, the search for how to increase relatedness to siblings $(r>0.5)$ has been a red herring; what is key is that average relatedness stays at 0.5 . Other red herrings include the possibility for eusociality to have evolved via cooperation between sisters (the parasocial route) because that would lead to $r<0.5(8)$ and the suggestion that high relatedness is a consequence rather than a cause of eusociality (15), in which case the observed correlation with monogamy would not be predicted (9). Finally, the monogamy hypothesis suggests that factors that facilitate monogamy, such as lifetime storage of sperm by females, are also important in explaining the distribution of eusociality.

\section{Get the Right Spite}

Inclusive-fitness theory explains altruism but also has a darker side. Spiteful traits, which are harmful to both actor and recipient, may be favored (4). Considering the classic two-party version of Hamilton's rule, if $c$ is positive (which is costly to the actor), and $b$ is negative (which is costly to the recipient), then $r b-c>0$ can be satisfied if relatedness $(r)$ is negative. Negative relatedness may seem a bizarre concept, but it simply means that the recipient is less related to the actor than is an average member of the population (SOM text).

Another way to think about spite is to distinguish between the primary recipient of the harming behavior (the individual physically attacked) and those secondarily influenced as a byproduct of this (those experiencing reduced competition from the harmed individual) $(16,17)$. From this perspective, spite can be favored if the actor is more related to the secondary recipients (who benefit) than to the primary recipients (who are harmed). Spite can therefore be thought of as altruism toward the secondary recipients: Harming an individual can be favored if this provides a
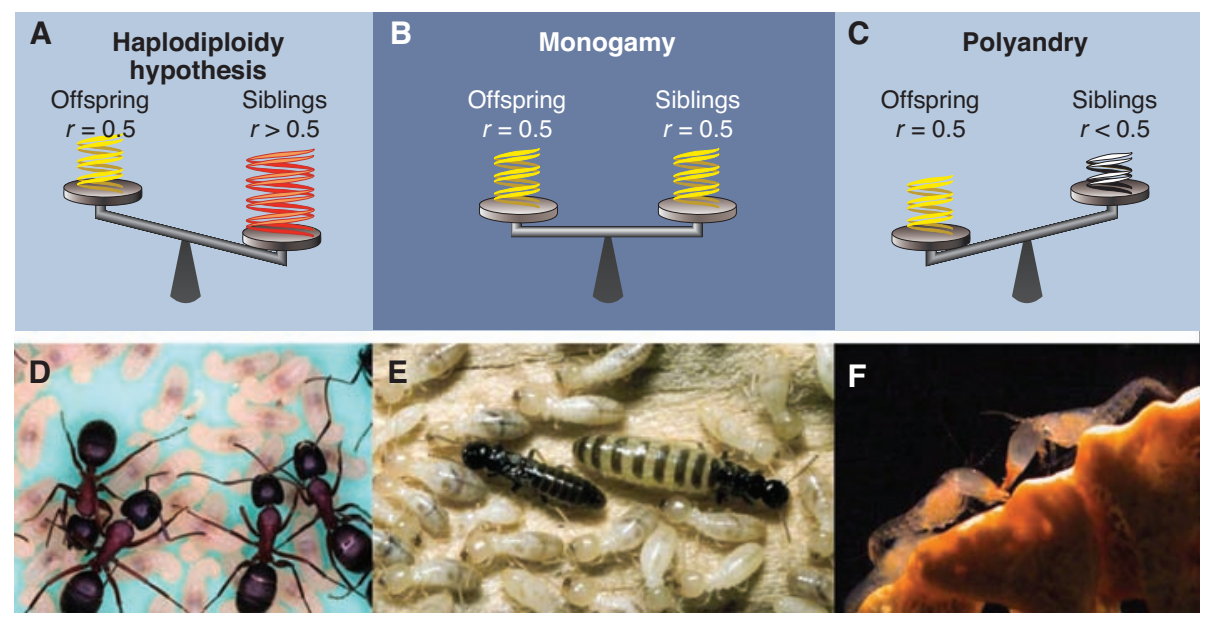

Fig. 3. Monogamy paves the way to eusociality. (A) The haplodiploidy hypothesis relies on individuals being more related to siblings than offspring, making siblings worth more than offspring. As originally envisioned, this appears to have been a red herring. (B) The monogamy hypothesis emphasizes that if an individual is equally related to its siblings and its offspring, even a very slight efficiency benefit for raising siblings translates into a selective advantage for helping. (C) Without strict monogamy, individuals are more related to their offspring than they are to their siblings so that a large efficiency benefit is required in order for sibling-rearing to be favored. (D) Sterile workers caring for brood in the ant Camponotus herculeanus. (E) A lifetime monogamous pair from the termite Reticulitermes flavipes. (F) Nonbreeding workers in the shrimp Synalpheus regalis. [Photos provided by David Nash, Barbara Thorne, and Emmett Duffy] 
benefit to closer relatives (Fig. 2C). These two different encapsulations of spite are different ways of looking at the same thing, using either a two- or three-party Hamilton's rule (SOM text) $(16,17)$. If the actor is more closely related to the secondary recipients than the primary recipients in a three-party rule, then this leads to a negative relatedness in the two-party rule.

Although many behaviors have been suggested as spiteful, they are usually explained as selfish behaviors that are costly to the recipient but provide a benefit to the actor $(c<$ $0)$ (Table 1). This benefit is often a reduction in future competition for resources, for the actor or their offspring, with confusion arising because the direct fitness consequences were only considered in the short term and not over the lifetime of the actor (SOM text) (18). What matters for natural selection are fitness consequences over the entire lifetime and not some arbitrary period. For example, herring gulls kill the chicks at neighboring nests, but this reduces the future competition over resources for both themselves and their offspring. Furthermore, there is no evidence that such examples involve a sufficient secondary benefit to relatives. Indeed, it has even been suggested that the conditions required are so restrictive that spite would be rare or nonexistent $(11,12)$.

However, recent theory has shown that a positive relatedness to secondary recipients can be obtained more easily than previously thought, suggesting that at least from a theoretical perspective spite is plausible (19) if there is (i) large variance in relatedness between competitors; (ii) kin discrimination, with harming behaviors aimed at individuals to whom the actor is relatively unrelated (making the actor relatively more related to the secondary recipients); or (iii) strong local competition so that harming the primary recipient provides appreciable benefits to secondary recipients. When these conditions are met, spiteful behaviors can be favored so as to reduce competition for relatives. Consider the extreme example of when two genetically identical (clonal) individuals are competing with a nonrelative to whom they are unrelated. One of the clone-mates could be selected to harm the nonrelative, at a cost to itself, if this harming reduces the nonrelative's ability to compete for resources and hence benefits the other clone-mate.

\section{Real Spite}

To demonstrate spite, it must be shown that a trait is costly to the actor (positive $c$ ) and costly to the recipient (negative $b$ ). In addition, for spite to have evolved by means of natural selection, the actor must be more closely related to the secondary recipients (beneficiaries) than to the primary recipient that they harm (Fig. 2C). The

to conspecifics that lack an immunity gene (22). In some cases, cell death is required to release the bacteriocins into the environment, and so it is clearly a costly trait (positive c). In addition, there is genetic linkage between the bacteriocin production and immunity genes so that close relatives both produce and are immune to a particular bacteriocin (22). Consequently, the release of bacteriocins kills nonrelatives, freeing up resources for clone-mates. Experimental work on bacteriocins has also supported the prediction that the relative advantage of spite is greater when variance in relatedness is higher (giving

sterile soldier caste in polyembryonic parasitoid wasps satisfies all these conditions (17). Females lay their eggs on the eggs of moth caterpillars, after which the wasp eggs divide asexually and consume the growing caterpillar from the inside (20). Although most larvae develop normally, a fraction become a soldier morph. Developing as a soldier is costly to the actor, because the soldiers are sterile, and costly to the recipient, because the soldiers seek out and kill other larvae within the host. Finally, soldiers preferentially kill larvae to which they are less related (those that have developed from other eggs), freeing up resources for their clone-mates.

Another example of spite is provided by the production of antimicrobial bacteriocins by many bacteria (21). These compounds are lethal
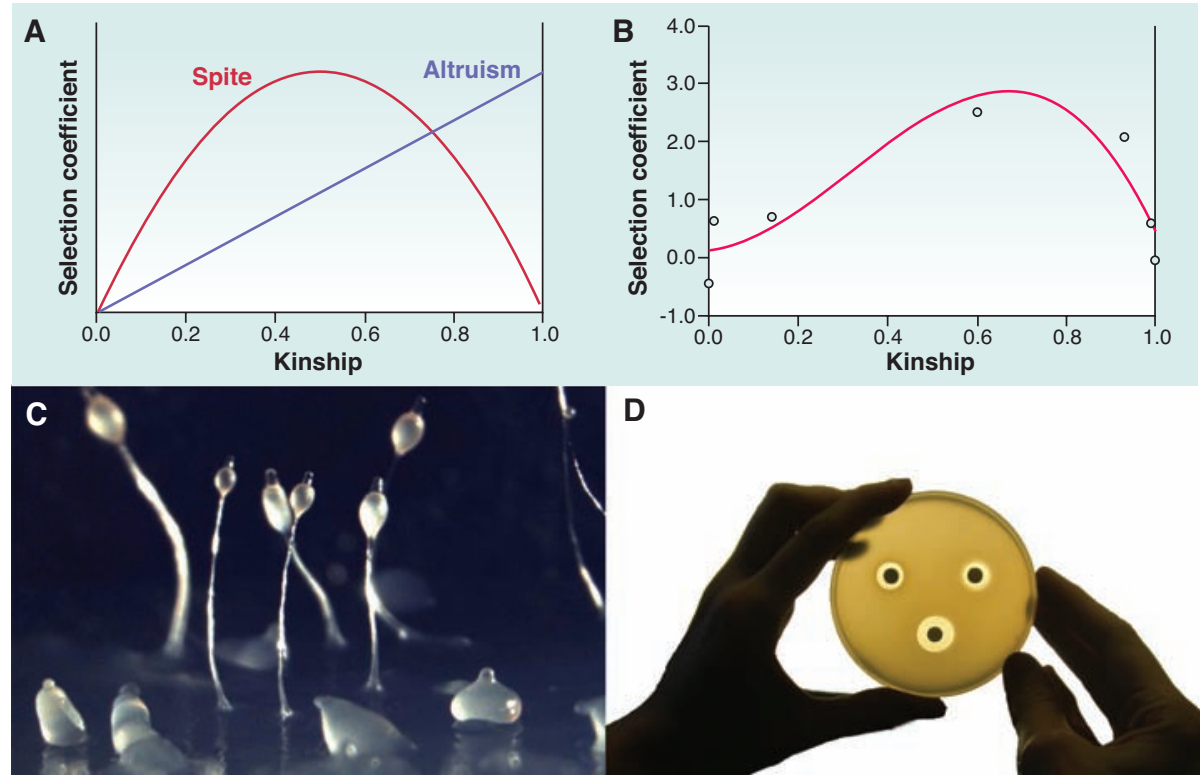

Fig. 4. Population structure, altruism, and spite. (A) Theory predicts that as relatedness within a patch increases, there is an increase in altruistic traits and a domed relationship with spiteful traits $(3,15)$. (B) As predicted, the relative benefit of bacteriocin production in Pseudomonas aeruginosa shows a domed relationship with relatedness (23) (C) Altruism: a fruiting body of the slime mold Dictyostelium discoideum. (D) Spite: A piece of filter paper soaked with a bacteriocin (left spot) causes an inhibition zone where bacteria cannot grow, in the same way as a traditional antibiotic (right spot; bottom spot shows both in combination). [Photos provided by Owen Gilbert, Margaret Riley, and Sara Cody] 
case can be rearranged so as to make a spiteful or altruistic two-party rule $(16,17)$.

However, there are also a number of reasons why it is useful to distinguish spite from altruism (17). From a behavioral perspective, there is a clear difference between helping and harming. Additionally, there are biologically interesting differences between altruism and spite. For example, local competition for resources typically selects for spite and against altruism (21); altruistic traits are predicted to show a positive relationship with relatedness (3), whereas spiteful traits are predicted to show a domed relationship (Fig. 4) $(19,21)$; and kin discrimination is key in the examples of spite, whereas altruism can often evolve without kin discrimination when limited dispersal keeps relatives together (3).

Spite can also be defined from a mechanistic or psychological perspective in primates and humans as a behavior that harms another or arises from a desire to harm another $(24,25)$. In these cases, harming is likely to be favored because on average it provides a direct benefit to the actor $(c<0)$ and is selfish, not spiteful. It is crucial not to mix definitions of spite by raising the problem and rarity of evolutionary spite then going on to examine a harming behavior that provides a direct benefit to the actor, and therefore is not evolutionary spiteful. The specific conditions required to favor evolutionary spite might make it relatively unlikely in humans and other primates. Indeed, spite may be rare in general because it is hard to obtain situations in which populations are structured so that harming nonrelatives is an efficient way of helping relatives.

\section{Greenbeards}

Hamilton $(3,26)$ pointed out that indirect-fitness benefits require genetic relatedness per se and not kinship. Imagine a gene (or cluster of tightly-linked genes) that gives rise to a conspicuous phenotype, uses this phenotype to discriminate between carriers and noncarriers of the gene, and leads to costly helping toward other carriers of the gene (Fig. 2D). This gene could be favored by natural selection, even if carriers share no other genes in common. Thus, the crucial requirement for altruism is genetic relatedness at the altruism locus and not genealogical relationship over the whole genome.

Dawkins proposed the hypothetical example of a gene that gives rise to a green beard while simultaneously prompting individuals with green beards to direct cooperation toward other green-bearded individuals (27). However, this "greenbeard" mechanism can also occur without a visible tag. What is required more generally is a single gene (or a number of tightly-linked genes) encoding both the cooperative behavior and causing cooperators to associate $(26,28)$. Greenbeards are one of the two ways in which natural selection can favor altruistic behavior, with the other being interactions with genealogical kin (3). However, despite this fundamental position it has been assumed that greenbeards would not occur in nature because they could be easily invaded by "falsebeards" (cheats) that displayed the beard without also performing the behavior (27).

This assumption has been overturned by the discovery of a number of altruistic greenbeards. In the slime mold Dictyostelium discoideum, individuals with the csa gene adhere to each other in aggregation streams and cooperatively form fruiting bodies while excluding noncarriers of the gene (5). Other examples have been found in yeast, a bacterial plant pathogen, and a lizard (SOM text). In addition, spiteful greenbeards have been discovered, including the $G p-9$ gene of the fire ant Solenopsis invicta (29). Workers with the $b$ allele at this locus use odor to determine whether prospective queens also carry this allele, dismembering them if they do not. Another example is provided by bacteriocin production, in which the bacteriocin-encoding gene and the immunity gene are tightly linked (22).

Theoretical work has clarified when we would expect greenbeards to occur (30). Greenbeards can be categorized into four groups, with different evolutionary dynamics, according to whether they are altruistic (helping) or spiteful (harming) and always expressed (obligate; for example, bacteriocins) or only expressed in response to the presence of the greenbeard in others (facultative; for example, $G p$-9). For all cases except the facultative altruistic greenbeard, the greenbeard is selected against at low frequencies and only favored when it has established itself to a certain frequency. Population structure can solve this problem by keeping individuals with greenbeards together. The best place to look for new greenbeards may be in microbes, in which asexual growth can lead to extreme population structuring; the relatively simple link between genotype and phenotype may prevent a decoupling of beard and social trait (falsebeards), and genetic knockouts facilitate the detection of greenbeards (30).

Some models for altruism in humans (31-34) and social insects (15) implicitly invoke greenbeard mechanisms without realizing this, such as the suggestion that altruistic individuals differ from individuals who are not altruistic in some observable characteristic [such as being more likely to smile and laugh $(31,32)]$ or models of "strong reciprocity" that assume punishment and altruism to be genetically linked $(33,34)$. However, there is no reason to suspect that traits such as smiling or punishment will be encoded by the same gene or closely linked genes as those that lead to altruism. Consequently, falsebeards could arise, and these proposed explanations for altruism would not be evolutionarily stable (30).

\section{Conclusions}

A unifying theme in all the issues that we have discussed here is the importance of the interplay between theory and data. The monogamy hypothesis cuts through the superfluous details by focusing on a key aspect of the underlying biology. Theoretical work on spite and greenbeards was spurred by biological examples but then led to a unifying framework that illuminated detection problems and suggested where to look for further examples. All of these examples illustrate that the distinction between genetics $(r)$ and ecology $(b / c)$ is both artificial and unhelpful. What really matters is how they interact, as has always been emphasized by Hamilton's rule.

\section{References and Notes}

1. C. Darwin, On the Origin of Species by Means of Natural Selection, or, the Preservation of Favoured Races in the Struggle for Life (John Murray, London, UK, 1859).

2. R. A. Fisher, The Genetical Theory of Natural Selection (Clarendon, Oxford, 1930).

3. W. D. Hamilton, J. Theor. Biol. 7, 1 (1964).

4. W. D. Hamilton, Nature 228, 1218 (1970).

5. D. C. Queller, E. Ponte, S. Bozzaro, ]. E. Strassmann, Science 299, 105 (2003).

6. A. Grafen, J. Evol. Biol. 20, 1243 (2007).

7. A. Gardner, Biol. Lett. 5, 861 (2009).

8. J. J. Boomsma, Philos. Trans. R. Soc. Lond. B Biol. Sci. 364, 3191 (2009)

9. W. O. H. Hughes, B. P. Oldroyd, M. Beekman, F. L. W. Ratnieks, Science 320, 1213 (2008).

10. R. L. Trivers, H. Hare, Science 191, 249 (1976).

11. R. Craig, Evolution 33, 319 (1979).

12. J. E. Strassmann, D. C. Queller, Proc. Natl. Acad. Sci. U.S.A. 104 (suppl 1), 8619 (2007)

13. J. J. Boomsma, Curr. Biol. 17, R673 (2007).

14. J. E. Duffy, K. S. Macdonald, Proc. Biol. Sci. 277, 575 (2010).

15. E. O. Wilson, B. Hölldobler, Proc. Natl. Acad. Sci. U.S.A. 102, 13367 (2005)

16. L. Lehmann, K. Bargum, M. Reuter, J. Evol. Biol. 19, 1507 (2006)

17. A. Gardner, I. C. W. Hardy, P. D. Taylor, S. A. West, Am. Nat. 169, 519 (2007).

18. K. R. Foster, T. Wenseleers, F. L. W. Ratnieks, Ann. Zool. Fenn. 38, 229 (2001).

19. A. Gardner, S. A. West, J. Evol. Biol. 17, 1195 (2004)

20. D. Giron, D. W. Dunn, I. C. W. Hardy, M. R. Strand, Nature 430, 676 (2004)

21. A. Gardner, S. A. West, A. Buckling, Proc. Biol. Sci. 271 1529 (2004).

22. M. A. Riley, J. E. Wertz, Annu. Rev. Microbiol. 56, 117 (2002).

23. R. F. Inglis, A. Gardner, P. Cornelis, A. Buckling, Proc. Natl. Acad. Sci. U.S.A. 106, 5703 (2009).

24. M. Hauser, K. McAuliffe, P. R. Blake, Philos. Trans. R. Soc. Lond. B Biol. Sci. 364, 3255 (2009).

25. K. Jensen, J. Call, M. Tomasello, Proc. Natl. Acad. Sci. U.S.A. 104, 13046 (2007)

26. W. D. Hamilton, in Biosocial Anthropology, R. Fox, Ed. (Wiley, New York, 1975), pp. 133-155.

27. R. Dawkins, The Selfish Gene (Oxford Univ. Press, Oxford, 1976)

28. L. Lehmann, L. Keller, J. Evol. Biol. 19, 1365 (2006).

29. L. Keller, K. G. Ross, Nature 394, 573 (1998).

30. A. Gardner, S. A. West, Evolution 64, 25 (2010).

31. R. H. Frank, Am. Econ. Rev. 77, 593 (1987).

32. M. J. Owren, J.-A. Bachorowski, in Emotions: Current Issues and Future Directions, T. J. Mayne, G. A. Bonanno, Eds. (Guilford, New York, 2001), pp. 152-191.

33. H. Gintis, J. Theor. Biol. 206, 169 (2000).

34. S. Bowles, H. Gintis, Theor. Popul. Biol. 65, 17 (2004).

35. G. R. Price, Nature 227, 520 (1970).

36. We thank ]. Alpedrinha, K. Boomsma, A. Griffin, M. Hauser, K. Jensen, L. Keller and P. Taylor for comments and the Royal Society, European Research Council, and Leverhulme Trust for funding.

Supporting Online Material

www.sciencemag.org/cgi/content/full/327/5971/1341/DC1 SOM Text

Tables $\mathrm{S} 1$ and $\mathrm{S} 2$

References

10.1126/science. 1178332 